\title{
Análisis del comportamiento micromecánico del asfalto
}

\author{
Micromechanical analysis of asphalt behaviour
}

\author{
Adriana de Jesús García \\ Lanamme-UCR, San José, Costa Rica, \\ ajesusgarcia23@gmail.com
}

Fecha de recepción: 03 de marzo de 2016 / Fecha de aprobación: 04 de abril de 2016

\section{RESUMEN}

Las recientes teorías clasifican el asfalto como una matriz continua, donde asociaciones de moléculas polares (asfaltenos y resinas) se dispersan en un fluido de moléculas de menor peso molecular y polaridad (aromáticos y saturados). Para entender mejor el comportamiento del asfalto se realizó una revisión de literatura, donde se pudo observar que la metodología más innovadora es la aplicación del microscopio de fuerza atómica, el cual permite caracterizar los materiales a escala nanomecánicas.

La técnica del AFM se ha venido utilizando en los últimos 20 años, como una de las herramientas que combina las potentes capacidades de la última generación de dispositivos de espectroscopía de fuerza atómica con toda la variedad de formación de imágenes disponibles en sus modos de medición: contacto, no contacto y fuerza pulsada. A raíz de esto, se han logrado obtener excelentes resultados de la morfología del ligante asfáltico, donde distintos investigadores han llegado a caracterizar un asfalto multi-fase definido como: la para-fase, la catana-fase, la perfase y la sal-fase. La revisión bibliográfica se realizó con el propósito de enriquecer el conocimiento acerca del comportamiento micromecánico del ligante asfáltico, lo cual servirá de base posteriormente para determinar y aplicar la técnica de indentación, la cual consiste en determinar el valor del módulo de rigidez. Por tanto el análisis del comportamiento micromecánico es una herramienta importante para las futuras investigaciones, las cuales deberían orientarse en el análisis de las propiedades del ligante asfáltico.

PALABRAS ClAVE: Comportamiento micromecánico, indentación, microscopio, fuerza atómica, módulo rigidez.

\begin{abstract}
Recent theories asphalt classified as a continuous matrix, which associations of polar molecules (asphaltenes and resins) dispersed in a fluid of molecules of lower molecular weight and polarity (aromatic and saturated). To better understand the behavior of asphalt a literature review, where it was observed that the most innovative methodology is the application of atomic force microscope, which allows to characterize materials nanomechanical scale was performed.
\end{abstract}

AFM technique has been used in the last 20 years as one of the tools that combines the powerful capabilities of the latest generation of devices with atomic force spectroscopy full range of imaging modes available on its measurement: contact, noncontact and down force. Following this, they have achieved excellent results in the morphology of the asphalt binder, where different researchers have come to characterize a multi-phase asphalt defined as: to stage the katana-phase, perfase and salt-phase. The literature review was conducted in order to enrich the knowledge of the micromechanical behavior of asphalt binder, which then serve as the basis for determining and applying the indentation technique, which involves determining the value of stiffness modulus. Therefore micromechanical behavior analysis is an important tool for future research, which should focus on analyzing the properties of asphalt binder.

KEYWORDS: micromechanical behavior, indentation, microscope, atomic force, stiffness modulus. 


\section{INTRODUCCIÓN}

El ligante asfáltico es una mezcla compleja de una gran variedad de moléculas que difieren en su forma, tamaño y composición química. Estas moléculas cambian con las condiciones de edad, de temperatura y de carga (Allen et al., 2012). El ligante asfáltico es un material de color negro a marrón, sólido o semisólido según la temperatura ambiente de los constituyentes predominantes, proveniente de la naturaleza o del procesamiento del petróleo (ASTM , 2013).

Para representar y explicar el comportamiento reológico del ligante asfáltico, generalmente se ha considerado la estructura como un sistema coloidal donde componentes polares de alto peso molecular (como son los asfaltenos) son disueltos en una matriz aceitosa de componentes no polares y de menor peso molecular llamados maltenos. Estos últimos son sustancias solubles y se subdividen en saturados, aromáticos y resinas con base en la composición y polaridad del material (Aguiar et al., 2015).

El artículo consta de una revisión bibliográfica acerca del comportamiento micromecánico del ligante asfáltico el cual ha sido caracterizado en los últimos años por diversos investigadores, quienes han utilizado la técnica del microscopio de fuerza atómica (AFM, por sus siglas en inglés), para caracterizar la composición química y microestructural del ligante asfáltico. El AFM es una herramienta sencilla, fiable y de fácil implementación que ha permitido a la tecnología moderna analizar el asfalto a escalas nanométricas y a diferentes formas dependiendo de la fuente de crudo de petróleo, historia térmica, y el método de preparación de la muestra (Carrión et al., 2010).

Con el presente artículo se busca analizar el comportamiento micromecánico del ligante asfáltico, mediante estudios previos de tal forma que se comprenda la técnica del microscopio de fuerza atómica y los principios del comportamiento del asfalto a escala micromecánicas.

\section{PRINCIPIOS Y TÉCNICAS DE AFM}

La técnica del microscopio de fuerza atómica (AFM) se desarrolló en 1986 por Bennig, Gerber, Stoll, Albrecht y Quate, en marzo de 1987 que fue aprobada definitivamente (Burnham y Colton, 1989). La técnica de nanotecnología ha sido una herramienta fundamental para caracterizar el comportamiento de asfalto (Nazzal y Qtaish, 2012). Además, está estrechamente relacionada con los métodos utilizados con aguja de perfilometría y el microscopio de efecto túnel (STM, por sus siglas en inglés). El AFM ha superado las limitaciones de la STM, dado que la señal emitida de este proviene de las variaciones de la corriente de efecto túnel y requiere que la superficie del material a ser analizado sea un semiconductor. Por tanto con el AFM las variaciones se dan entre la punta y los átomos de la superficie que se está analizando pues la interacción entre estos son los que generan la señal de voltaje (Binnig et al., 1987).

El funcionamiento básico de AFM para el cual fue diseñado es medir la fuerza generada entre una punta afilada que está sujeta a una viga en voladizo y al material a ser evaluado. Sondeando la superficie de la muestra mientras que va recogiendo los datos (Howland y Benatar,
2000). La Figura 1 muestra el microscopio de fuerza atómica (AFM) que posee el LanammeUCR, el cual ha sido utilizado por investigadores de esta institución.

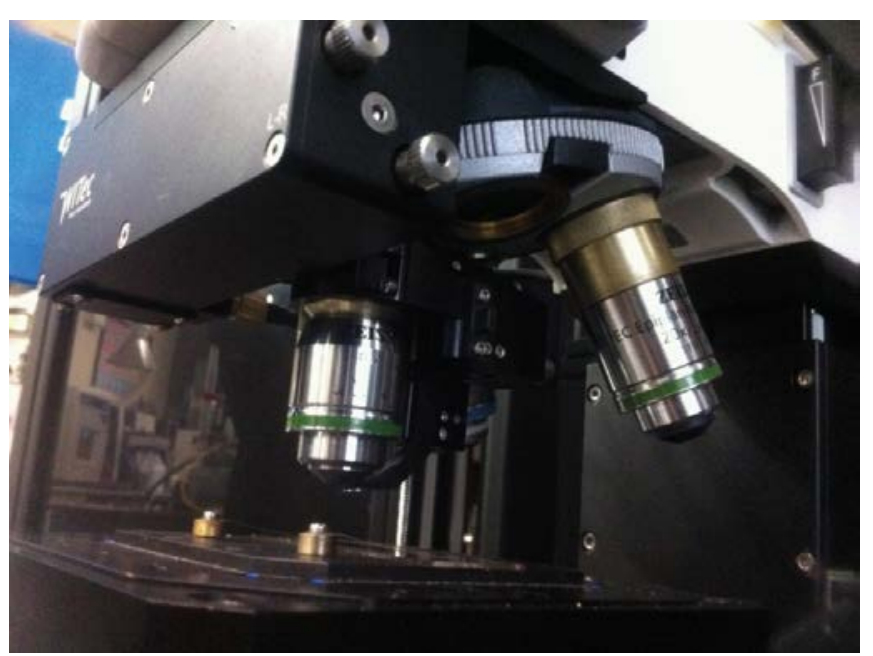

Figura 1. Microscopio de Fuerza Atómica (Tomada de: LanammeUCR).

El AFM requiere de una interacción entre todas sus partes, las cuales son: el voladizo, el fotodetector de 4 cuadrantes, la punta, el piezoeléctrico y el láser tal como se muestra en la Figura 2. Dicho funcionamiento consiste en que la punta realice un barrido de la muestra y pueda determinar la topografía y las propiedades micromecánicas del material en estudio, mediante desviaciones del láser en el fotodetector. Para llevar a cabo la medición el equipo se encuentra sujetado a una mesa antivibratoria y dentro de una cámara diseñada para reducir la interacción del entorno (Aguiar et al., 2015).

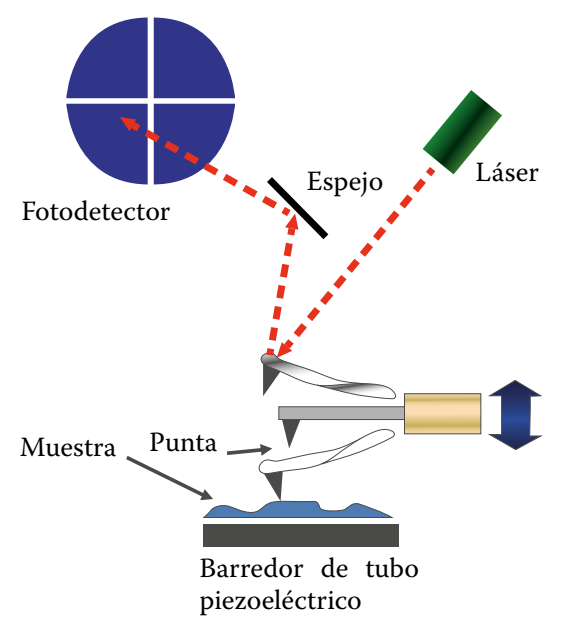

Figura 2. Esquema del funcionamiento de AFM.

(Tomada de: instituto de ciencia y tecnología de polímeros, 2013).

El microscopio de fuerza atómica (AFM) es un instrumento que permite medir las propiedades mecánicas del ligante asfaltico con resolución atómica. De tal forma que se obtiene de esta forma información importante de la muestra, las cuales influyen a escala macroscópica, tales 
como la rigidez, la viscoelasticidad, la plasticidad, la adhesión, la fractura y la curación (Binnig et al., 1986). La principal aplicación del AFM ha sido la formación de imágenes superficiales a alta resolución de las estructuras biológica del material (Burnham y colton, 1989).

\section{MODOS DE MEDICIÓN}

Para la obtención de las propiedades AFM cuenta con tres modos de medición, los cuales son: modo de contacto ( $\mathrm{CM}$, por sus siglas en inglés), modo de no contacto (AC, por sus siglas en inglés) y modo de fuerza pulsada o contacto intermitente (Raghavan, VanLandingham, y Nguyen, T., 2000).

El modo de contacto es la interacción entre la punta que se une al final del voladizo con una baja constante de resorte y la constante de resorte efectiva que mantienen los átomos de la muestra. Debido a que la punta barre la superficie, la fuerza de contacto origina la deflexión del voladizo de modo que este se adapta a la superficie topográfica de la muestra, permitiendo distinguir zonas con diferentes comportamientos mecánicos, para esto la fuerza entre la punta y la muestra se mantienen constante (Haugstad, 2012).

En el modo de no contacto (AC), la fuerza aplicada es mucho menor que para el $\mathrm{CM}$, lo cual es una ventaja cuando no se quiere deteriorar la superficie a medir y cuando se estudian muestras blandas o elásticas tales como el ligante asfáltico (Raghavan, VanLandingham y Nguyen, 2000). En el modo AC las fuerzas entre la muestra y la punta son muy pequeñas, por lo que sus mediciones se basan en la frecuencia resonante o amplitud del voladizo (Haugstad, 2012).

Por tanto, en el modo de fuerza pulsada o contacto intermitente, la punta realiza una interacción de contacto intermitente con la muestra mientras va barriendo en la superficie, evitando de esta forma el deterioro de la muestra que puede resultar de operar en el modo de contacto (Haugstad, 2012). Este modo permite ampliar las capacidades del microscopio más allá de la simple medición de la topografía, de tal forma que se puede obtener las propiedades tales como: la rigidez local, la adhesión, la viscosidad, la disipación de energía, el tiempo de contacto y las fuerzas de largo alcance. Esto se puede hacer a tasas normales de exploración, dado que el sistema trabaja a velocidades de miles de píxeles por segundo (Haugstad, 2012).

La electrónica del modo de fuerza pulsada incluye un sistema de adquisición de datos de alta velocidad, un generador de modulación libremente programable y un módulo de evaluación de datos en tiempo real. Con el almacenamiento de la medición completa, se lleva a cabo una amplia evaluación de datos post-procesamiento.

De lo anterior se deriva que dependiendo del modo de operación y la rigidez del material bajo investigación, se debe elegir el modo más adecuado para la medición. En la Tabla 1 se sintetizan las ventajas y desventajas de la técnica AFM.

\section{OTRAS TÉCNICAS DE ANÁLISIS}

Para caracterizar las propiedades micromecánicas del ligante asfáltico, se pueden utilizar otras técnicas de análisis que permitirán tener un detalle completo del desempeño del ligante a escala micromecánica. De lo anterior se deriva la determinación del análisis químico y micromecánico del ligante asfáltico, donde se han venido implementando varias técnicas que permiten caracterizar la composición del ligante asfáltico. Dentro del análisis de la composición química se tiene: la Espectrometría Infrarroja por Transformada de Fourier (FTIR, por sus siglas en inglés), espectroscopía Raman, difracción de rayos-X, cromatografía de gases acoplada a espectrómetros de masa, espectrofotometría ultravioleta (UV, por sus siglas en inglés), cromatografía líquida de alto desempeño (HPLC, por sus siglas en inglés), cromatografía de columna, cromatografía de capa fina con detección de ionización de llama conocido comercialmente como Iatroscan y espectrometría de masas con fuente de plasma acoplado inductivamente (ICP-MS, por sus siglas en inglés). Dentro de las técnicas que pueden ser utilizadas para estudiar

\section{VENTAJAS Y DESVENTAJAS DE AFM}

\section{Tabla 1. Ventajas y desventajas que presenta AFM en sus modos de medición.}

\begin{tabular}{|c|c|c|}
\hline Modo de trabajo & Ventajas & Inconvenientes \\
\hline Contacto & $\begin{array}{l}\text {-Altas velocidades de barrido } \\
\text {-Resolución atómica }\end{array}$ & $\begin{array}{l}\text {-Fuerzas laterales que pueden distorsionar la imagen. } \\
\text {-Fuerzas normales (fuertes atómica). } \\
\text { - Daño a las muestras blandas }\end{array}$ \\
\hline No contacto & $\begin{array}{l}\text {-No se aplica fuerza sobre la muestra. } \\
\text {-Útil para muestras hidrofóbicas. }\end{array}$ & $\begin{array}{l}\text { - Menor resolución lateral. } \\
\text { - Menor velocidad de barrido. }\end{array}$ \\
\hline Contacto pulsado o intermitente & $\begin{array}{c}\text {-Mayor resolución lateral } \\
\text {-Menor interacción con la muestra. } \\
\text {-Mejor obtención de propiedades micromecánicas. }\end{array}$ & $\begin{array}{l}\text { - Menor velocidad de barrido. } \\
\text { - Aplicación de mayor fuerza daña la muestras blandas. }\end{array}$ \\
\hline
\end{tabular}

(Fuente: instituto de ciencia y tecnología de polímeros, 2013). 
el comportamiento micromecánico del asfáltico incluyen: el reómetro dinámico de cortante (DSR, por sus siglas en inglés), el analizador mecánico dinámico (DMA, por sus siglas en inglés), el microscopio de fuerza atómica (AFM) y la microscopía por barrido electrónico (Aguiar et al., 2015; Allen et al., 2012). La aplicación y desarrollos de las técnicas antes mencionadas servirán para realizar una evaluación exhaustiva en el comportamiento de los ligantes asfáltico. Si bien es cierto AFM es una excelente herramienta en lo respecta a análisis micromecánico, la comparación y correlación con el análisis químico es muy importante.

\section{COMPORTAMIENTO MICROMECÁNICO DEL ASFALTO}

Con el objetivo de comprender los principios del comportamiento micromecánico del asfalto, se ha realizado una recopilación de información a partir de investigaciones previas, las cuales se han desarrollado utilizando la técnica del AFM. Uno de los primeros estudios científicos fue desarrollado por Loeber, Sutton, Morel y Mulleer, (1996); que obtuvieron imágenes de baja resolución que según indican son moléculas de asfaltenos dentro de un gel o matriz asfáltica. Así mismo observaron imágenes de estructuras tipo "abeja" distribuidas aleatoriamente dentro de la matriz asfáltica. Por su parte, Pauli, Branthaver, Robertson y Grimes (2001) demostraron a través del AFM que las asociaciones de asfaltenos o partículas coloidales junto con grupos funcionales polares pueden resultar en el desarrollo de funciones microestructural en la interfase ligante-aire.

En un estudio similar, Jäger, Lackner Eisenmenger, (2004) y Masson, Leblond, Margeson, (2007), revelaron que la morfología del asfalto está dividida en el área que corresponde a la estructura abeja, permitiendo observar la clasificación del asfalto en 3 grupos distintos: 1) dispersión fina $(0,1-0,7 \mu \mathrm{m})$ en matriz homogénea, 2) dominios de $0,1 \mu \mathrm{m}$ y 3 ) cuatro fases diferentes de distintos tamaños (catana, per, para y salfase). Sin embargo, tuvieron la limitante que no lograron correlacionar la morfología por AFM y los cuatro componentes del asfalto (saturados, asfáltenos, resinas y aromáticos).

En cambio Allen, Little y Bhasin, (2012), enfatiza que previo al envejecimiento, los ligantes asfálticos muestran dos fases distintas: una fase continua y otra fase dispersa. Mediante la nano indentación determinó las propiedades micromecánicas tales como: rigidez, adherencia y comportamiento elástico-plástico. Los investigadores demuestran que la influencia del envejecimiento oxidativo genera cambios microestructurales en la estructura del asfalto, tales como: la dispersión de fases, el agrupamiento y la materialización; por lo que sugieren que las estructuras tipo abeja son el resultado del último proceso, donde una nueva subfase es identificada dentro de la fase dispersa.

Por su parte, Dourado, Simao y Leite, (2012) mediante el AFM mostraron las diferencias significativas en la elasticidad del asfalto, tanto en estructura tipo abeja como en la fase continua. Ellos realizaron indentaciones en distintos puntos de la estructura tipo abeja y se encontró que la recuperación elástica depende de la estructura coloidal de la abeja. McCarron, Yu, Tao y Burnham, (2012) asumen que las estructuras tipo abeja están relacionadas a las ceras en los asfaltos, concluyendo que un incremento en la temperatura puede ser asociado con una reducción en la altura de las estructuras tipo abeja. Los autores basan este supuesto en investigaciones previas por Moraes, Pereira, Simao y Leite, (2010), quien hipotetizó que las estructuras tipo abeja son el resultado de la cristalinización de ceras dentro del ligante asfáltico.

La microscopía de fuerza atómica (AFM) se puede utilizar para determinar los módulos de relajación de distribuciones bimodales y trimodales de microfases de asfalto para evaluar las diferencias entre el comportamiento viscoelástico (Allen, Little, Bhasin y Lytton, 2013).

Siguiendo los resultados del comportamiento del asfalto Yu, Burnham y Tao, (2015), recomiendan comparar lo obtenido mediante AFM con otras mediciones a nanoescala tales como nanoindentación o análisis mecánico dinámico. De esta forma las relaciones entre las microestructuras, las propiedades mecánicas y químicas del ligante asfáltico se podrían establecerse, para desarrollar un modelo relacionado con la estructura de las propiedades mecánicas.

Otros investigadores han utilizado varias técnicas de AFM para estudiar los efectos de mezclas tibias (WMA, por sus siglas en inglés) en la nanoestructura y la microestructura, así como las propiedades adhesivas y cohesivas de un aglutinante de asfalto. Estos observaron que las estructuras tipo abeja están presentes en asfalto originales y modificados, (Nazzal, Abu-Qtaish, Kaya, y Powers, 2015.).

Finalmente, Aguiar et al., (2015), mediante la técnica de AFM lograron determinar las distintas fases presentes dentro de los distintos componentes del asfalto. Las mediciones obtenidas parecen indicar que en el caso del asfalto evaluado, las estructuras tipo abeja no están relacionadas con los asfaltenos, por tanto la hipótesis que las estructuras tipo abeja son el resultado de procesos de oxidación, modificación del asfalto con polímeros o cristalización de ceras parafínicas dentro del ligante asfáltico parece no ser factible. Sin embargo, si hay evidencia clara que el envejecimiento por temperatura tiene un efecto sobre la morfología del asfalto, y como tal, dentro de cada una de las fases presentes en los distintos componentes SARA (saturados, resinas y aromáticos).

Para representar la información antes mencionada por investigadores que han estudiado la estructura del asfalto; se muestran la Figura 3, en esta se puede observar: la topografía, la fuerza máxima, la rigidez y la adhesión presentes en el ligante asfáltico, para la obtención de estas propiedades se utilizó el modo de fuerza pulsada. Las mediciones fueron obtenidas mediante un análisis morfológico del asfalto PG64-22, el cual proviene de la refinería Costarricense de Petróleo (RECOPE), dicho análisis tuvo lugar en las instalaciones de los laboratorios de micromecánicas del LanammeUCR. 


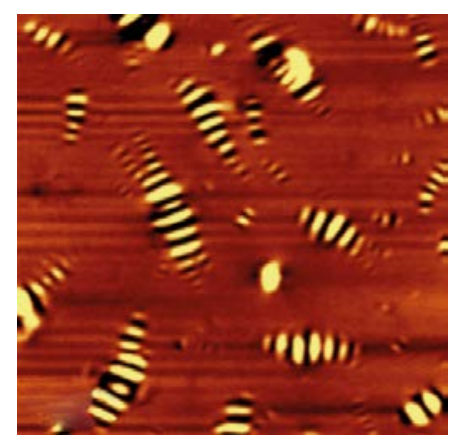

(a)

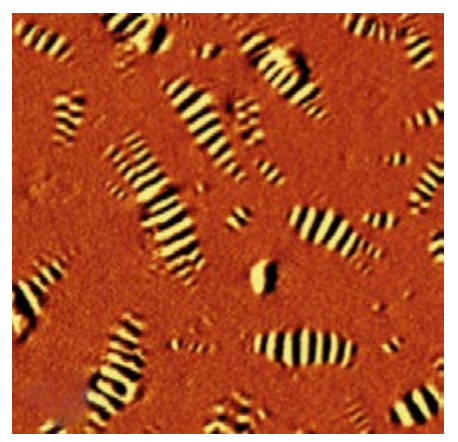

(b)

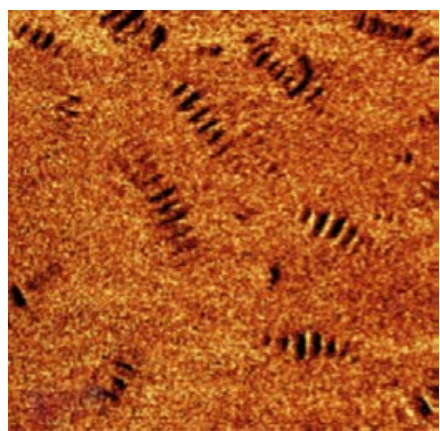

(c)

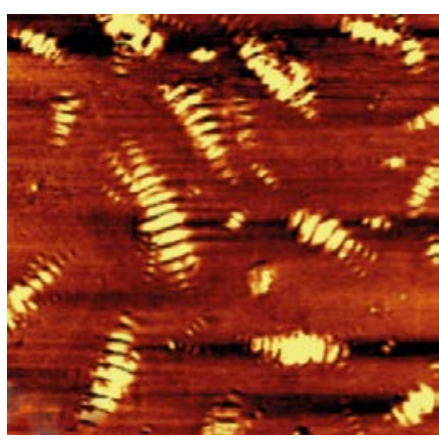

(d)

Figura 3. Mediciones en el modo de fuerza pulsada, asfalto PG64-22: a) topografía, b) fuerza máxima, c) rigidez y d) adhesión. (Fuente: LanammeUCR)

\section{CRITERIOS DE DISCUSIÓN}

Durante mucho tiempo diversos investigadores han realizado la labor de estudiar el comportamiento micromecánico del asfalto, pues es a partir de este análisis que se puede mejorar los modelos de desempeño de la mezcla asfáltica. A raíz de esto se ha venido utilizando el AFM para determinar las propiedades físicas y micromecánicas del ligante asfáltico, entre ellas están: la penetración máxima y la fuerza relacionada a esta, la adhesividad, el trabajo por adhesión, la rigidez y la respuesta viscoelástica, (Dourado et al., 2012). Los resultados de topografía, fuerza máxima, adhesión y rigidez son las propiedades fundamentales obtenidas con el microscopio de fuerza atómica en el modo de fuerza pulsada o contacto intermitente. Estas permiten visualizar el comportamiento del ligante asfaltico cuando se le está aplicando determinada fuerza, que tan rígido $o$ adhesivo resulta ser.

De lo anterior se derivan las distintas teorías acerca de las propiedades micromecánica del asfalto, las cuales han llevado a los investigadores a determinar las cuatro fases fundamentales en el asfalto que se denominan catana-fase (estructura tipo abeja), peri-fase (alrededor de la catanafase), para-fase (regiones disueltas) y sal-fase (zona de más alto contraste) (Aguiar et al., 2015).

La determinación de las fases del ligante asfáltico ha generado un debate entre los científicos dado que cada uno tiene distinta hipótesis de la estructura tipo abeja. Tal es el caso de Masson, Leblond y Margeson, (2006) quienes planteaban que la peri-fase es la región oscura que rodea a los asfaltenos, y que esta fase se debía a las resinas que son los compuestos más polares de los maltenos y de menor dureza que los asfaltenos. Por su parte, Aguiar et al., (2015) afirman que los asfaltenos no están asociados directamente a la catana-fase; mientras tanto, Pauli, Grimes, Beemer, Turner y Branthaver, (2011) brindan una nueva teoría de que las ceras eran las responsables de la estructura tipo abeja, concluyendo así que la interacción entre las ceras de parafina y los componentes restantes que constituyen el asfalto son los responsables de la microestructura del mismo, incluyendo la estructura tipo abeja. Por tanto, Jäger et al., (2004) y Aguiar et al., (2015) coinciden en que las estructuras tipo abeja observadas en la superficie del ligante asfáltico se caracterizan por un aumento de la penetración de la punta del AFM en el ligante e indica que las partes más rígidas de la matriz están interconectadas y que una posible explicación viene dada por la disposición espacial de las micelas. Por otro lado Yu., et al (2015) expresa que la microestructura de los ligante asfáltico se desarrolla dependiendo de las fuentes de crudo, el método de preparación de la muestra, y la historia térmica.

La nanoindentación es un campo de conocimiento que día a día avanza a un ritmo muy rápido especialmente con el microscopio de fuerza atómica, dado que proporciona resultados más rápidos y precisos. Esta técnica es una excelente herramienta para estudiar el movimiento individual de los átomos en las estructuras del material a analizar (Roa, Oncins, Díaz, Sanz, y Segarra, 2011).

Como parte de los resultados que ha obtenido en LanammeUCR referente a las fases del ligante asfáltico, se muestra en la Figura 4 la topografía y composición de fases (catana, per, para y sal-fase) que envuelven a la matriz asfáltica tal como lo detalla Aguiar (2015). Lo anterior demuestra la hipótesis de que las estructuras tipo abejas están presente en la catanafase, la cual se encuentra en el grupo de los maltenos.

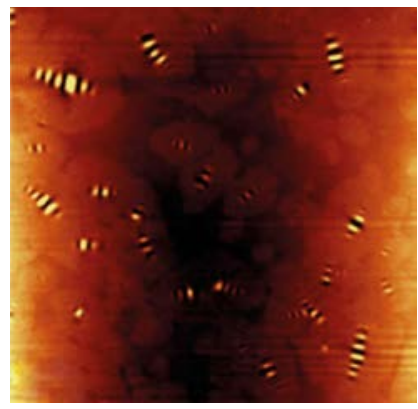

(a)

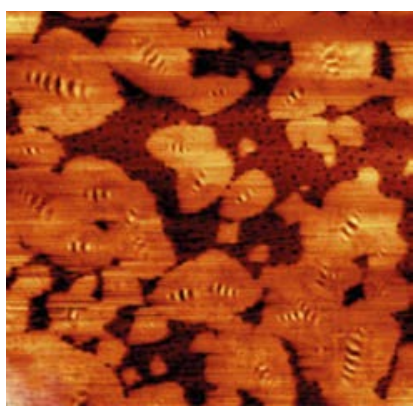

(b)
$3 \mu \mathrm{m}$

Figura 4. Imágenes de mediciones en el modo de no contacto (a) topografía y (b) detección de fase del asfalto original. (Fuente: LanammeUCR) 


\section{CONCLUSIONES}

Mediante la revisión bibliográfica consultada se logró estudiar a fondo las técnicas del microscopio de fuerza atómica, el cual no solo permite obtener la morfología de la superficie, sino también propiedades como la topografía, la rigidez y adhesión. El conjunto de estas propiedades se obtiene con el modo de fuerza pulsada, por tanto, la selección del modo va a depender de lo que se desea obtener.

Con base en las investigaciones consultadas, se puede concluir que el comportamiento microestructura del asfalto tipo abeja no están relacionadas con los asfaltenos, dado que estos se encuentran en la para-fase brindando de esta forma la rigidez del material; mientras que los maltenos son los que brindan mayor adhesividad, lo que es congruente pues son componentes más viscosos.

Lo anterior sirve de fundamento para futuras investigaciones como la determinación del módulo de rigidez en el ligante asfaltico. Para esto se requiere aplicar técnicas de nanoindentación la cual permite estudiar los rangos de deformación elástica del material. La información de dichas técnicas puede ser correlacionada con los resultados de variables obtenidas con otras metodologías de laboratorio.

\section{REFERENCIAS}

1. Aguiar, J.P., Salazar, J., Bonilla, V., Rodríguez, E., Leiva, F y Loria, L. (2015). Morphological analysis of asphalt phases using AFM. Road Materials and Pavement Design, Vol. 16, pp. 138-152.

2. Allen, R., Little, D y Bhasin, A. (2012). Structural Characterization of Micromechanical Properties in Asphalt Using Atomic Force Microscopy. Journal of Materials in Civil Engineering. Vol. 24 (10), pp. 1317-1327 Obtenido de: http://ascelibrary.org/doi/ pdf/10.1061/(ASCE)MT.1943-5533.0000510

3. Allen, R., Little, D., Bhasin, A y Lytton, R. (2013) Identification of the Composite Relaxation Modulus of Asphalt Binder Using AFM Nanoindentation. Journal of Materials in Civil Engineering, Vol. 25, pp. 530-539.

4. ASTM D8-13b. Standard Terminology Relating to Materials for Roads and Pavements. ASTM International, West Conshohocken, PA, 2013.

5. Binnig, G., Quate, C.F. y Gerber, C. (1986). Atomic force microscope. Phys. Rev. Lett. 56., 930-933.

6. Burnham, N. A., Dominguez, D. D., Mowery, R. L., y Colton, R. J. (1990). Probing the surface forces of monolayer films with an atomic-force microscope. Phys. Rev. Lett., 64 (16), 1931-1934.

7. Burnham, N.A., y Colton, R.J. (1989). Measuring the nanomechanical properties and surface of materials using an atomic force microscope. J. Sci. Technol. A 7., 2906-2913.

8. Carrión, M. S., Valbuena, J.A y Gomez, H. J. (2010). Dispositivo interfase digital para la integración del modo de imagen en espectroscopio de fuerza monomolecular. Consejo Superior de Investigaciones Cientificas.

9. Dourado, E.R., Simao, R.A y Leite, L.F.M. (2012.). Mechanical properties of asphalt binders evaluated by atomic force microscopy. Journal of Microscopy, Vol. 245, pp. 119-128. .

10. G. Binnig, CH. Gerber, E. Stoll, T. R. Albrecht y C. F. Quate. (1987). Atomic Resolution with Atomic Force Microscope.

11. IOPSCIENCE, Europhys. Lett., 3 (12), pp. 1281-1286.

12. Haugstad, G. ( 2012). Atomic Force Microscopy: Understanding Basic Modes and Advanced Applications. John Wiley \& Sons, Inc. Hoboken, NJ.

13. Howland, R., y Benatar, L. (2000). A practical guide to scanning probe microscopy. Recuperado de: <http://raman.plmsc.psu. edu/ manias/MatSc597/docs/pract_guie.pdf) (May 2010).

14. Roa, J., Oncins, G., Díaz, J., Sanz, F y Segarra, M. (2011). Calculation of Young's Modulus Value by Means of AFM. Recent Patents on Nanotechnology, 5, 27-36. 
15. Jäger, A., Lackner, R., Eisenmenger-Sittner, C y Blab, R. (2004). Identification of Microstructural Components of Bitumen by Means of Atomic Force Microscopy (AFM). Proc. Appl. Math. Mech., Vol. 4, pp. 400-401. .

16. Loeber, L., Sutton, O., Morel, J., Valleton, J.M y Muller, G. (1996). New direct observations of asphalts and asphalt binders by scanning electron microscopy and atomic force microscopy. Journal of Microscopy, Vol. 182, Issue 1, pp. 32-39.

17. Masson, J., Leblond, V y Margeson, J. (2006). Bitumen morphologies by phase-detection atomic force microscopy. Journal of Microscopy, Vol. 221, pp. 17-29.

18. McCarron, B., Yu, X., Tao, M y Burnham, N. (2012). The Investigation of “Bee-Structures" in Asphalt Binders. Qualifying Project. Worcester, MA.

19. Moraes, M.B., Pereira, R.B., Simao, R.A y Leite, L.F. (2010.). High temperature AFM study of CAP 30/45 pen grade bitumen. Journal of Microscopy, Vol. 239, Issue 1, pp. 46-53.

20. Nazzal, M., Abu-Qtaish, L., Kaya, S y Powers, D. (2015.). Using Atomic Force Microscopy to Evaluate the Nanostructure and Nanomechanics of Warm Mix Asphalt. Journal of Materials in Civil Engineering. Nazzal, M.D y Qtaish, L.A. (2012). The Use of Atomic Force Microscopy to Evaluate Warm Mix Asphalt. Report FHWA/OH- 2012/19. Athens, OH.

21. Pauli, A.T., Grimes, R.W., Beemer, A.G., Turner, T.F y Branthaver, J. F. (2011). Morphology of Asphalts, and asphalt fractions and model wax-doped asphalts studied by atomic force microscopy. International Journal of Pavement Engineering, Vol. 12, pp. 291-306.

22. Raghavan, D., VanLandingham, M., Gu, X., y Nguyen, T. (2000). Characterization of hetero geneous regions in polymer systems using tapping mode and force mode atomic force microscopy. Langmuir, 16(24), 9448-9459.

23. Rodriguez, C., (2013). Microscopía de Fuerza Atomica (AFM), modos de operacion y aplicaciones. Instituto de Ciencia y Tecnologia de Polimeros.

24. Yu, X., Burnham, N y Tao, M. (2015). Surface microstructure of bitumen characterized by atomic force microscopy. Advances in Colloid and Interface Science, pp. 17-33. 